\title{
Religious covenant: interpretation of faith in the utilitarian rationality- alliance between divine right and human right
}

\author{
Zhihui Yang \\ College of Foreign Languages and Cultures of Sichuan University, Chengdu 610065, China \\ tsyankee@163.com
}

Keywords: religious covenant; federal theology; puritans; reason and logic.

\begin{abstract}
Religious covenant of New England, availing secular reason and logic, made a cultural reformation of the traditional theology, and framed the ideological foundation of the federal theology by systemizing the belief of "original sin", "covenant of works", "covenant of redemption", and "covenant of grace". By indulging in grace form God, religious covenant suspended the God's supreme power, personified and secularized divinity; meanwhile, it sanctified secular reason, logic and consciousness of self-determination. Essentially, religious covenant coalesced divine and secular rights, redefined the cultural and spiritual identity of the New Englanders. Fabricated in the historical and social settings of commercialization, religious covenant supplied a subverted reinterpretation of traditional religion thought, and articulated the acclamations for reason and logic and the commercial principles of laisser-faire and fair-play in the early capitalist society.
\end{abstract}

\section{新英格兰的宗教契约：理性对信仰的实用主义解读一神权和人权结盟}

\section{杨智慧}

四川大学外国语学院, 成都610065, 中国

关键词：宗教契约; 联邦神学; 清教徒; 理性和逻辑

摘要：新英格兰的“宗教契约”是以世俗理性和逻辑对传统神学的人文化革新，它将“原罪说”、 “行为契约”、“救赎契约”、“恩典契约”有机地结合起来成为联邦神学的主要思想。新英格兰 宗教契约以神的恩典悬置了神的至高地位，把神“人格化”，把神学“世俗化”，同时把世俗的 理性、逻辑、自决“神圣化”，它是是人权和神权的结盟、是新英格兰的清教徒移民民族世俗 身份和宗教精神身份的文化界定。宗教契约的思想体系在近代逐渐开始商业化的社会背景中 形成, 新英格兰联邦神学思想是对传统宗教观念的颠覆性阐释, 这种解读既体现了资本主义 社会对思想的理性和自由的诉求, 也折射出了资本主义经济发展中对商业自由（laisser-faire） 和公平 (fair play) 原则的推崇。

\section{1. “异端”学说对新英格兰“正统”清教思想的挑战}

清教主义是一套完整的神学观念体系, 虽然它盛行与 17 世纪, 尤其是在北美殖民地之一的 新英格兰，但是其思想体系却既不发端于 17 世纪也不源自于清教徒，而是自文艺复兴以来欧 洲大陆宗教思想和人文思想发展、演变的产物。它是革新思想和守旧思想的混合体, 它执着 地依附于传统思想 (“tenacious grip upon the past”)， “清教徒的虔诚显现出原始基督教的复活

(“a resurrection of primitive Christianity”) ; (2)同时, 清教神学观又吸收了人文主义的思想成 分。新英格兰的文化思想和这些被称为联邦神学的清教契约思想是紧密结合的, 这种结合事 实上实现了 17 世纪新英格兰世俗自由思想和宗教思想的集成化和一体化。联邦神学契约思想 
体系整合了“行为契约”（Covenant of Works）、“救赎契约”（Covenant of Redemption）、“恩 典契约”（Covenant of Grace），并且成为了美国后来资本主义伦理精神的基本内涵。

清教徒的联邦神学思想是十七世纪加尔文主义的系统化, 是借用经院哲学中理性和信仰相 结合的方法, 对宗教信条进行的革新, 目的是弥补原始信条在理性质问下的某些逻辑断裂和 缺失。而这些教条逻辑上的内聚力不足甚至是矛盾往往是各种“异端”学说反抗“正统”思想的 理据。例如, 阿米尼乌斯派（Arminianism）和反律法派（Antinomianism）, 这两派都认为加 尔文教的教条所规定的道德是极端主义的, 认为加尔文教派的思想提出加尔文主义无视道德 的主动性, 它所宣扬的神秘的“命定论” (predestination) 由于过分地抹杀了人的主体性, 无益 于促进普通信徒的道德积极性; 适得其反的是, 对于意志薄弱的民众, 出于人性固有的弱点 和对是否被神恩 “拣选” 的怀疑, 加尔文派所坚持的道德极端主义和命定论会成为他们懒散放 纵的借口。

于是，阿米尼乌斯宣称：“恩典的救赎功效取决于人的意志，人根据天赋的自由有权力接 受或拒绝神的恩典”。这种“异端”思想中关于人的“主体独立”和“自决权”的意识在17世纪的 正统清教徒听来是对神权大逆不道的櫭越。但是如果认为人关于自身主体性的观念是仅仅是 文艺复兴和宗教改革的功绩就有失偏颇了, 事实上人的主体性意识在希伯来人的宗教中就已 经埋下了思想种子。用叙事学的观点来看, 在《旧约圣经》中, 当神用直接引语式说“你不可 杀人”（thou shall not kill）的时候, 就已经是对人主体性的一种确定了。也就是说, 神和人之 间这种主体和客体的关系促使希伯来人意识到其自身也有独立的主体性, 这种主体性蕴含着 人的尊严独立和道德自律。换句话说, 关于神的思辨衍生出了人的自我观念和意识, 希伯来 人相信神赐予了他们道德的自决权, 因此, 人人都能够自己判别善恶, 也有权选择善。 ${ }^{(4}$ 正 是这种主体意识给 17 世纪的新英格兰清教徒“异端”带来了反抗“正统”理论和思想。所以, 在 阿米尼乌斯派的学说中, 人作为理性和自由的主体, 是主动的、自决的, 不一定必须是是神 来拣选人, 而也有可能是人选择接受或拒绝神的恩典。而反律法派则也以上帝的无限恩典为 由而摈弃了摩西的律法; 伯拉纠派 (Pelagianism) 则更加激进, 他们甚至否定了原罪学说, 断言不需要神的恩典, 人只要靠自己的努力就可以走向救赎之路。这些“异端”的涌现表明世 俗的理性和自由意志正在逐步地壮大, 给崇尚和维护神权的正统清教思想带来了巨大的挑 战。

\section{2. 恩典契约：世俗理性对宗教信仰革新}

清教徒的世界是神布置设定的, 是稳固的、有限的、不变的。这个世界的一切对其而言都 是合乎理性的、可理解的；清教徒是有思想的保守分子，时刻警惕着以“革新”为旗号的异端 学说; 他们心满意足地生活在神与他们约定的世界, 深信“谋事在人, 成事在天” (Man proposes and God disposes）。面对“异端”的出现, “谋事在人”促使清教徒行动起来维护他们的信仰, 同时也就是维护清教思想所设定的特定权利秩序。“正统”清教徒认为“异端”的出现是有神意 的, 虽然“异端”学说挑起争论，但是通过争论消除“异端”, 就更能彰显“真理”。为此, 清教 徒认为有必要把“正统”的神学思想进行拓展, 通过契合逻辑和理性的思辨, 把一些新的观念 或未被阐释的学说囊括在其联邦神学体系之内, 可于是对恩典契约的思想进行全新的阐释和 再解读就势在必行了。

恩典之约是神与选民 (罪人) 所立的约, 不同于最初神和亚当之间约定的行为契约, 注重 行为的道德性和纯洁性，恩典契约的思想核心是“因信称义”（justification by faith）:

“在律法之下, 神要人交出义来, 在恩典之下, 他将义赐给人; 律法是与摩西和行为相连 的, 恩典是与基督和信心相连的; 律法祝福好人, 恩典拯救罪人; 这个试验的要点, 不再以 遵守律法作为得救的条件, 只是在乎接受基督与弃绝基督;律法是靠行为称义 (justification by works），而恩典则是靠信心称义。”

“因行称义”向“因信称义”的过渡, 是宗教禁锢衰落、尘世生活崛起的体现, 是传统神学向 理性人文的妥协。恩典契约本质上是法制性的, 体现了十七世纪习惯法的精神, 是宗教改革 
家和法学家的思想联盟。但神权的这种妥协在激进的“异端”看来是不够的, 这体现在“异端” 和“正统”对恩典理解的差异：唯信仰论者认为无需恪守摩西的律法，只要“虔信”，神的恩典 就会降临; 虔诚把神的恩典（grace of God）就成为无条件的恩典（free grace）。而阿米尼乌 斯主义者则进一步提出, 如果信仰无法带来救赎, 那就让理性去寻找救赎。唯信仰论者只强 调虔诚, 而阿米尼乌斯论者只注重理性, 可在正统的清教思想中这两者是一个综合体, 是不 可分割的, 而联邦神学则是这一综合体的完美阐释体系; ${ }^{\circledR}$ 这也体现了“正统”清教思想家是中 世纪经院哲学思想的继承者。针对上述两派 “异端”的学说, 恩典契约要解决的问题一方面是 要让人们有道德的责任感, 另一方面则是要人们坚定获得恩典救赎的信心。如果神的律法要 继续树立, 人们必须知道他们在神的救赎计划中的作用; 而要让人们对救赎有信心, 就必须 让他们相信被救赎是有条件约定的, 并且神也是遵守这些条件的, 神不会任意地践踏或撕毁 这些条约。这样, 十七世纪的清教徒就冒着对上帝的神圣和全能不敬的风险, 用尘世中人 的理性和道德着手把他们的神理性化、人格化, 让神纡尊降贵地进入并遵守俗世的契约, 这 样才能平息信仰和理性的矛盾, 达成神权和人权的“契约”。但是, 清教徒并不认为神被他们“人 化”并束缚于世俗的契约中了, 相反，他们把神权和人权的契约看成是世俗理性对“神启”深入 思索和积极解读的结果, 看成是神的恩典, 是神对人博爱的进一步拓展和深化。

恩典契约要完成的其实是神权对人权管理方式的巨大转变。如果说行为契约注重的是人的 行为, 那么恩典契约则注重的是人的信念, 这就更加强调人要有完美的心灵而不是完美的行 为, 尽管人在行为上有缺点, 但只要他有正直和向善的心, 就可得救赎。行为契约下神检查 人的行为因而审判人, 而恩典契约下, 神感知人心灵的信念进而滕顾人, 神由行为契约中令 人恐惧的惩罚者, 变成了恩惠契约中人心灵的安慰者。①(日)就是说, 清教徒通过恩典契约用 人性改造了神性, 用人的律法限制了神的权力; 不再是神禁锢了人的自由, 而是人限制了神 的权柄, 以“神为本”变成了以“人为本”。通过让宗教从对人行为的繁琐规定转向对人思想和 心理的指引, 联邦神学实现了一种更便捷但更有效的宗教和社会的管理模式; 但反过来, 从 行为的监管到心理的引导本身就是对人身一种解放, 是人类理性对自由索求的初战告捷。联 邦神学所诉求的身体的解放和对理性的推崇后来直接推动了世俗社会中自由和民主意识的 发展, 虽然这是联邦神学先驱们始料未及的, 因为新英格兰的清教领袖们绝不是社会自由和 民主的支持者。(11)传统的教条来看，人性随着人被逐出伊甸园而永远地“邽落了”，所以行 为契约成了清教徒“罪蕉的胎记”和受惩罚的“紧䈐咒”; 但从历史的角度来看, 其实是理性启 蒙趋于普遍化了。17世纪的新英格兰充斥着来自欧洲大陆的民主自由思想, 清教徒的“紧䈨咒” 亟待摘取, 或者说一场世俗的思想解放运动势在必行, 而宗教契约就是这场解放运动的理论 诉求。于是, 宗教契约极大地放松了神权对人性的牵制, 用神的恩典和人的虔信为依托, 为 清教徒规划了一座受神应许并受神护佑的世俗的伊甸园（city upon the hill）; 在这里世俗的 人和“邽落的”人性被宽容, 给本来因为罪䔣和值落而内疚、对拯救感到绝望的人许诺了新的 救赎希望。清教徒之所以被救赎, 是因为他们的心灵时刻都谨守着一个约定, 而且在为这个 约定不解奋斗; 只要这种奋斗不息, 就没有什么罪责可以把他们拉向沉沦。因此, 信仰完全 是一个意志问题，恩典契约建立的根基不是神的威严，也不是人的道德，而是人的意向。(12) 于是, 尘世生活不再是放荡的、罪莩的、充满痛苦的, 世俗生活成了神赐予清教徒的第二次 机会, 世俗社会成了他们争相向神展现其道德、勤劳和善良人性, 并争取被救赎的检验场。 他们在这里将“苦我心智, 劳我胫骨, 不谋私利, 共赴大难, 成功因天恩浩荡, 失败则甘愿受 诛”。13这是清教联邦神学对传统宗教的彻底颠覆, 它把人的自由意志提高到了无以复加的高 度, 神代表的再也不是最高的原则和权威了; 因为神的律法通过契约被理性化和人性化了, 所以无需再盲目地畏惧神的律法; 比起神泪茫, 清教徒更热衷于具体而清晰的世俗理性和逻 辑。理性带来的意志自由, 即使是在地狱投射的阴影下, 也显得那么地诱人。 


\section{3. 行为契约、“原罪”观念、救赎契约：恩惠契约的逻辑预设和理性综合}

清教神思想宣称: 神许可契约随着时空的变更而相应发生变革, 第一次变革是由于人的道 德内省而发生的, 第二次则是通过基督的福音而进行的; 而神之所以允许契约的变革, 是因 为他宽容了人的懦弱本性。(14)表面看好像是神对世人的启示有一个特定的秩序, 其实这是联 邦神学认识论的逻辑设定，是宗教契约的一个前提，有了这个前提就可以把“原罪说”、“行为 契约”、“救赎契约”、“恩典契约”结合成一个完整的联邦神学思想体系。起初, 神创造了亚当 并和他签订契约, 如果亚当遵守神所规定的行为, 神就赐给他和他的子孙永生, 这个契约强 调人要以行为被神所悦纳, 因此被称作“行为契约”。可是, 世俗的人终究无法拔除其肉体和 欲望的“劣根”而使人有所堕落, 因此, 人永远无法完全遵守行为契约, 所以原罪感和对神惩 罚的恐惧感一直是索绕清教徒的哥梦, 这种忧患和困惑是清教神学思想家首先要思考并安抚 的。要安抚这种自行为契约以来基督徒的罪䔣、恐惧和绝望感, 就要把其心灵和肉体的双重 禁锢从基督教传统教义中解放出来。此外, 17世纪人文主义兴起, 理性思维对信仰频频质疑。 要维持宗教的不容置疑的权威, 新英格兰的联邦神学家就必须从世俗的理性出发对神学契约 论思想进行合乎逻辑的再阐述和再解读, 目的是要“软化”（soften）其宗教教义严苛, 抽象化 信条的公义内涵, 15解除人们来自亚当原罪的伦理包䘞, 并使他们相信世俗的道德是获得救 赎的途径和希望。

“原罪说”和“行为契约”是统摄基督徒身心的“达摩克利斯剑”，必须拔除，也就是说“原罪意 识”和“行为律法”必须得以合乎理性的缓解和释放。首先, 清教徒对原罪观念从契约论角度进 行了理性的再解读：他们认为“原罪”是他们天生就欠神的一份债，但是仁慈的“债主”对他们 以再定新契约的方式做出了妥协和让步, 只要他们遵守契约就可走出罪的羁绊。在新契约的 观念中，虽然“原罪”在宗教伦理上仍是清教徒道德和自由意志的根本障碍，但是“原罪”并没 有剥夺他们的理性和意志的自由, 理性和自由意志是上帝赋予他们的, 人人与生俱来的, 只 要用理性去理解契约, 用意志去执行契约, 人就可以摆脱罪的束缚而走向自由和救赎。这样 联邦神学思想不仅把没有把原罪看成是人类无法摆脱的劣根, 相反, 它原罪转化成了激发人 用理性和意志进行思考和采取行为进而赎免原罪的动力。所以, 清教徒没有像中世纪的基督 那样在忓悔中忽视和贬低世俗生活, 而是积极主动地在世俗生活中用理性和自由意志在尘世 中自强不息以获取神的悦纳，并最终为自己在天国中争得一席之地。

接下来还需要在原罪说、行为契约和恩典契约之间有一个逻辑的过渡或关联, 还需要一个 逻辑链条, 即“救赎契约”, 来把这些契约形成一个思想体系, 进而强化宗教契约思想的内在 逻辑。恩典契约是人和神之间的约, 而救赎契约则是神和基督之间的约, 也就是说, 神不仅 向世人许诺而且也向他自己许诺遵守恩典契约, 这样旧约中耶和华神权的任意性受到了限 制。显然, 基督的设定是清教徒释放原罪感和摆脱行为契约的充要条件, 基督的牺牲既弥补 了世人的罪, 也替人履行了行为契约; 正如行为契约中亚当的罪作为原罪被归于他的后代子 孙一样, 基督的救赎则除去了世人的原罪枷锁。基督的出现既实现了神的威严和公义, 也彰 显了神恩的浩荡, 而清教徒只需要遵守全新的契约, 用虔诚和道德争取神的悦纳。恩典契约 的前提是“信”（faith）; 人内心的顺服和虔诚才是最终救赎依据, 外在的行为表现只是一种 必要的附条件, 没有宗教虔诚的世俗行为即使是高尚的德行也不能换得救赎。“虔信”, 不只 是一种简单的信仰行为, 还是契约签订的基本条件, 它在清教徒内心规定着一个行为的义务, 并要求这种内在的义务转化为外在的行为; 所以, 拒绝接受恩典契约的“自然人” (natural men) 将仍然按照行为契约的规定接受审判, 他们仍然必须遵守摩西严格的律法, 而进入新约定 的人将不必在惧怕人性的弱点会使他们沉沦, 因为即使沦了只要能以宗教的虔诚不断地自 拔, 就不会丧失被救赎的希望和机会。可见联邦神学对“正统”清教徒和所谓不接受恩典契约 的“自然人”以及“异端”是区别对待的, 这既排除了异己的教派, 又颠覆了加尔文“救赎命定” 的思想，可称得上一种宗教的民主化改革了。

联邦神学中原罪、行为契约、救赎契约和恩典契约被纳入到了一个整体, 形成了一个完美 的逻辑体系, 这个逻辑体系是世俗理性的非凡创造。如果有人戏虐说新英格兰清教领袖把基 
督教的神学思想玟渎成了世俗的“联合股份公司”，吕那么，这个股份公司的经营原则就是人 的理性和逻辑, 这个世俗化的宗教思想体系也延伸到了社会管理观念中, 并成为新英格兰世 俗政治思想的“参考文献”和建立世俗政府的行动指南。

\section{4. 清教徒宗教信仰的民主化和实用化}

恩典契约最重要的是给了人们道德的选择权并要求人们道德责任自负, 赋予人的理性充分 的自由, 让人做自己道德的主宰者。也可以说, 恩典契约提供给清教徒一个和神在理性和逻 辑上讨论救赎条件的机会, 并最终实现了神权和人权之间的部分移交和两者在公平公正的基 础上的融洽合作, 并极大地激发了清教徒世俗德行的主动性。恩典和虔信完全源自于神, 然 而这一切却是通过世俗逻辑思维和理性论辩而得以彰显的, 因此是所有清教徒能够充分理 解、认知和接受的。于是, 人便从对神的完全的恐惧和无条件服从中解放了, 他们把接受或 拒绝神恩的权利视为人合乎理性的权力。这种信仰彻底自由化的主张（laisser-faire）在17世 纪的清教徒那里被提出, 既令人惊呀但又合乎情理, 因为清教徒在欧洲大陆已经历经了宗教 改革浪潮和理性启蒙思想的洗礼。从此, 契约让人从神那里接过了道德自查和责任自负的权 利, 走向救赎的天堂还是走向沉沦的地狱, 都成了人自己的选择: “成功因天恩浩荡, 失败则 甘愿受诛”。这样，契约论就解构了加尔文主义的命定论，把神的拣选和人的沉沦理性化了, 这是联邦神学思想最伟大的成就：自由、民主、平等。契约中神权和人权协商并结盟, 信仰 对理性的实施了神圣化而理性对信仰的进行了世俗化。

新英格兰的联邦神学用“有条件契约”（conditional covenant）取代了加尔文教义中的“有条 件拣选” (conditional election) 或“命定救赎”; 派瑞·米勒对于这种变更的最终结论是：新英格 兰神学领袖把恩惠契约变成了赤裸裸的实用主义法则; 换句话说, 只要遵守契约, 任何人都 可以被拣选; 他们用世俗的辩证逻辑修订了加尔文的神学思想, 清教徒们保留了新教基本思 想的同时，树立了他们对理性、逻辑和艺术的信仰。18这种修订不止是出于宗教心理的主观 愿望, 更多是出于 17 世纪经济、政治和社会的因素。新英格兰清教徒通过契约把人的救赎用 对神的虔诚为条件确定了下来, 清教徒只要虔诚信奉, 并不断有意识地自我改善, 神就应当 赐予他们天国的救赎, 这是一种公平的交易, 正如世俗中的商业交易一样, 必须坚持公平原 则（faire play）; 新英格兰的清教徒是实用主义的门徒, 他们不会轻易放弃自己的权利, 即 使是面对和上帝契约。19所以, 派瑞-米勒感叹说, 加尔文的鬼魂会愤怒地谴责恩典条约把对 基督教的信仰变成了一种“精神的商业买卖”，而且这场交易必须谨遵世俗的公平原则。

清教徒认为自己是亚伯拉罕的子孙, 因而也被纳入了亚伯拉罕和神的契约中, 只是清教徒 不仅坚持自己的理性, 而且把这种世俗的、人的理性也赋予了神, 尽管他们仍然坚持神是超 越人的理性的。既然神也被以法律的方式列入了契约, 成了具有和人一样遵守尘世律法的主 体, 那么神就不仅仅是口头承诺, 而是要承担义务。和中世纪历史观一样, 他们认为神与世 人之间契约的确立、呈现、演变、贯彻执行, 就是人类历史的全部过程和意义, 但不同于中 世纪观念的是他们认为除了契约中规定的责任之外, 神不会更多地参与世俗的事务。(20清教 徒以赋予神“人格”和“法律责任”的方式，把神权的任意、全能、全知、全在巧妙地悬置起来， 而悬置的理由是神对人的恩典, 这样在对神威的敬畏和对神恩的感谢中, 清教徒实现了自己 的解放。清教契约思想把经院哲学的逻辑、理性和文艺复兴的自由思想兼收并蓄, 发挥到了 极致, 在信仰和理性之间创建了一个稳固的阿基米德点: 法律和契约。最终, 民主意识、契 约精神和商业自由原则（laisser-faire）和公平的原则在联邦神学思想中实现了完美的结合。

\section{5. 结语}

联邦神学的思想虽然只是发声于新英格兰地区的弹丸之地, 但其影响却是极其深远的, 它 是经院哲学、文艺复兴和启蒙运动三者结合后在新英格兰清教徒神学观念中的总爆发, 是宗 教改革思想的进一步深化和拓展, 是世俗理性思想的极大解放。它把人和神的关系从旧式的 
身份关系变成了契约关系, 并把这种契约关系扩大到人和人的整个社会关系中去了。它标志 着人类从对自然力量盲目畏惧和羁绊中的进一步解放, 自由的理性逐渐替代信仰的必然; 人 类意识逐渐从对任意的、非理性的、神秘的、由非可知力量控制的自然物质秩序的无意识的、 盲目的崇拜中逐渐苏醒, 这是世俗理性和世俗权利意识的苏醒。于是, 一个新的、更理性化 的秩序也随之在主动选择、自愿契约、责任自负、自由自决等世俗意识的基础上建立起来了。 这也是建立在身份观念上的封建等级意识向以自由契约为基础的宪法权力意识的过渡。当神 和人之间的必然关系都被契约关系所取代的前提下，世俗社会中人和人之间的不平等的身份 关系就更是封建等级制难以维系的了。这样, 宗教契约不仅确立了人与上帝之间的关系, 还 把平等契约的意识拓展到世俗社会中人与人之间的关系, 以及人与社会组织 (如政府) 之间 的关系，所有这些关系的共同思想基础是：权利、平等和自由。

\section{References}

(1)(2)(5): Miller, Perry. The New England Mind: the Seventeenth Century, Harvard University Press, 1971, p.365, p368, p.366.

(4): Perry, Marvin. An Intellectual History of Modern Europe, Houghton Mifflin, 1992, p.8.

(6): Scofield, Cyrus I, et al., Li Chenzhong translation, "Secretary for the blessing of the Bible to be released" Shanghai: Canton Association book, 1951, pp.319-320

(7)(8): Miller, Perry. The New England Mind: the Seventeenth Century, Harvard University Press, 1971, p.374, p.371, p.373.

(10): "Thus, we may cease being apprehensive over His hidden terrors, He will abide by definite regulations '...he is so compassed about with his nature and property, and Covenant, that he hath no liberty to strike" "ibid. p. 380 .

(11): ibid. p.120, p. 378.

13): Zhao Yifan, "Criticism of American Culture", Beijing: Sanlian Bookstore, 1994, p.122

(14): “...God had allowed the covenant to grow with time, first dispensing it through conscience, and now through Christ, the preaching of the Word.... He has done this out of delicate consideration for human weakness." Miller, Perry. The New England Mind: the Seventeenth Century, Harvard University Press, ibid. p.382.

(15) (16): ibid. pp. 405, 386.

(17): : ...blasphemous degradation of the tripartite divinity into a joint stock company" ibid. p.407.

18: " "...the final outcome in all the New England preaching of the covenant theory was shamelessly pragmatic injunction.” ibid. p.395.

(19): “... for by binding God to the terms,.... No puritan was going to surrender himself, even to God, without getting something in return." ibid. p.389.

(20) "God never does anything for His people unless 'he doth it by virtue of, and according to his Covenant"” ibid. p378. 